\title{
Avaliação de medidas de controle de flebotomíneos
}

\author{
Evaluation of sandfly control measures
}

\author{
Kárin Rosi Reinhold-Castro ${ }^{1}$, Regiane Bertin de Lima Scodro', \\ Alessandra de Cassia Dias-Sversutti ${ }^{2}$, Herintha Coeto Neitzke ${ }^{2}$, Robson Marcelo Rossi ${ }^{3}$, \\ João Balduíno Kühl' ${ }^{4}$, Thaís Gomes Verzignassi Silveira ${ }^{4}$ e Ueslei Teodoro ${ }^{4}$
}

\begin{abstract}
RESUMO
Realizou-se coletas de flebotomíneos de maio de 2005 a abril de 2006. Os resultados foram comparados com os das coletas realizadas entre abril de 2001 e setembro de 2002, para avaliar as medidas empregadas para diminuir a densidade destes insetos, no Recanto Marista, município de Doutor Camargo, Estado do Paraná. As coletas foram feitas com armadilhas de Falcão, em domicílios e galinheiros, das 22 às 2 horas, semanalmente, quatro vezes ao mês. Em 2005 e 2006, coletaram-se 213.195 flebotomíneos, 1.113,8 em média por hora (MH) e em 2001 e 2002, 199.821 (MH=1.653,5). Nyssomyia neivai prevaleceu (75,4\%) em todos os ecótopos que, juntamente com Nyssomyia whitmani (23,4\%), Migonemyia migonei (0,8\%) e Pintomyia fischeri $(0,4 \%)$, representaram $99,7 \%$ do total coletado. Nos ecótopos representados por galinheiros foram coletados $88,7 \%$ dos flebotomíneos. Constatou-se que houve queda na densidade de flebotomíneos no período de coletas 2005 e 2006 em relação à de 2001 e 2002 , especialmente no domicílio.
\end{abstract}

Palavras-chaves: Leishmaniose tegumentar americana. Nyssomyia neivai. Nyssomyia whitmani. Flebotomíneos. Controle.

\begin{abstract}
Collections of sandflies were made between May 2005 and April 2006. The results were compared with those from collections undertaken between April 2001 and September 2002, in order to evaluate the measures used to decrease the density of these insects in Recanto Marista, municipality of Doutor Camargo, State of Paraná. The collections were carried out by Falcão traps inside domiciles and hen sheds, from 10 p.m. to 2 a.m. once a week, four times a month. In 2005 and 2006, 213,195 sandflies were collected (average of 1,113.8 per hour), compared with 199,821 (average of 1,653.5 per hour) in 2001 and 2002. Nyssomyia neivai predominated (75.4\%) in all the ecotopes. Nyssomyia neivai, Nyssomyia whitmani, Migonemyia migonei and Pintomyia fischeri accounted for $99.7 \%$ of all the sandflies collected. The ecotope of hencoops accounted for $88.7 \%$ of the sandflies collected. It was observed that the sandfly density had decreased between the 2001-2002 and 2005-2006 collections, especially in homes.
\end{abstract}

Key-words: American cutaneous leishmaniasis. Nyssomyia neivai. Nyssomyia whitmani. Sandflies. Control.

A incidência da leishmaniose tegumentar americana (LTA) vem crescendo na América Latina, principalmente no Brasil, onde foram registrados 605.062 no período de 1980 a $2005^{18}$. Destes casos, 13.384 foram notificados na Região Sul, dos quais 13.206 $(98,7 \%)$ ocorreram no estado do Paraná ${ }^{18}$.

O controle de flebotomíneos tem sido realizado com o emprego de inseticidas químicos em paredes de casas, anexos (abrigos de animais e silos) $)^{78121321}$ e impregnados em mosquiteiros ${ }^{491014}$. Contudo, a descontinuidade no uso de inseticidas e/ou o uso em épocas inapropriadas têm limitado o sucesso desta medida ${ }^{12410}$. Outro fator que dificulta esse método de controle é a precariedade das habitações ${ }^{10}$. Somando-se ao fato desses insetos não serem estritamente associados ao domicílio, cessado o efeito residual dos inseticidas os mesmos voltam a freqüentar este ambiente ${ }^{16}$. A utilização de inseticidas nos ambientes silvestres representa um elevado risco de dano ao meio ambiente ${ }^{416}$, pois pouco se conhece sobre a localização dos criadouros de flebotomíneos ${ }^{16}$. Além disto, o tamanho das áreas a serem desinsetizadas torna o método impraticável do ponto de vista ambiental e pelo custo muito elevado ${ }^{16}$.

Medidas alternativas de controle, que possam ser incorporadas na rotina das populações que vivem em áreas de risco podem

\footnotetext{
1. Análises Clínicas, Universidade Estadual de Maringá, Maringá, PR. 2. Ciências da Saúde, Universidade Estadual de Maringá, Maringá, PR. 3. Departamento de Estatística, Universidade Estadual de Maringá, Maringá, PR. 4. Departamento de Análises Clínicas, Universidade Estadual de Maringá, Maringá, PR. Apoio Financeiro: Fundação Araucária (Convênio 225/2005).

Endereço para correspondência: Drª Kárin Rosi Reinhold-Castro. R. Jouji Nakamura 61, Jardim Novo Horizonte, 87010-110 Maringá, PR.

Tel: 5544 3025-2333/9988-6584; Fax: 5544 3261-4860

e-mail: karindecastro@yahoo.com.br

Recebido para publicação em: 07/12/2007

Aceito em: 05/06/2008
} 
reduzir a freqüência de flebotomíneos no domicílio e peridomicílio, diminuindo a incidência da $\mathrm{LTA}^{36}$. A atratividade desses insetos por aves (galinhas) tem sido pesquisada e comprovada em diversos estudos experimentais, demonstrando que os galinheiros constituem barreiras zooprofiláticas, diminuindo sua densidade no domicílio e o seu contato com o homem² 23272829 .

No Recanto Marista, a primeira pesquisa foi realizada para conhecer a fauna e frequiência de flebotomíneos no domicílio e em abrigos de animais domésticos no peridomicílio e para avaliar as condições ambientais ${ }^{27}$. Nessa localidade vem sendo realizado o acompanhamento de medidas que foram introduzidas para diminuir a população desses insetos no peridomicílio e evitar o acesso dos mesmos ao domicílio ${ }^{25} 28$. Estes autores verificaram que as medidas introduzidas, apesar de executadas parcialmente, reduziram consideravelmente a população desses dípteros no domicílio. Este trabalho representa a terceira avaliação da influência da reorganização e limpeza do peridomicílio e a desinsetização de edificações e abrigos de animais domésticos no município de Doutor Camargo, Estado do Paraná, como forma alternativa de controle de flebotomíneos.

\section{MATERIAL E MÉTODOS}

A pesquisa foi realizada numa área de lazer (Recanto Marista), às margens do rio Ivaí, com 57,6 hectares, dos quais 40,8 cobertos por mata do tipo floresta estacional semidecidual que, apesar de ter sofrido grandes alterações, ainda é habitada por aves e mamíferos silvestres. O Recanto Marista situa-se no município de Doutor Camargo ( $52^{\circ} 13^{\prime}$ Longitude Oeste e $23^{\circ} 33^{\prime}$ Latitude Sul), que se insere na mesorregião norte central do Paraná ${ }^{27}$.

Durante o período de coletas de flebotomíneos, a temperatura foi medida no início (22 horas) e no final (2 horas) de cada coleta. A temperatura média no período de maio de 2005 a abril de 2006 foi de $16,2^{\circ} \mathrm{C}$; com a mínima de $5,8^{\circ} \mathrm{C}$, em julho de 2005 e máxima de $31,9^{\circ} \mathrm{C}$, em janeiro de 2006 . A precipitação média anual de chuva no período de 1996 a 2004 foi de $1.809 \mathrm{~mm}$; a menor precipitação do período foi em 1999 (1.413mm) e a maior em 1997 (2.150mm). Em 2005, a precipitação de chuva foi de $1.937 \mathrm{~mm}$ e, em 2006, nos meses de janeiro a abril as precipitações totalizaram $952 \mathrm{~mm}$. Os dados de precipitação pluviométrica foram fornecidos pela Cooperativa Agroindustrial (COCAMAR), unidade de Doutor Camargo e pela Estação Climatológica da Universidade Estadual de Maringá (UEM).

As coletas de flebotomíneos foram feitas com 11 armadilhas luminosas de Falcão, em diferentes ecótopos (Figura 1), semanalmente, quatro vezes ao mês, das 22 horas às 2 horas, de maio de 2005 a abril de 2006, num total de 192 horas em cada armadilha, exceto a armadilha 9 (ecótopo 9) que falhou na quarta coleta do mês de março. Nesta armadilha, foram 188 horas de coletas. 0 horário das coletas foi o mesmo dos trabalhos anteriormente realizados no Recanto Marista ${ }^{252728}$. As armadilhas foram instaladas da seguinte forma:

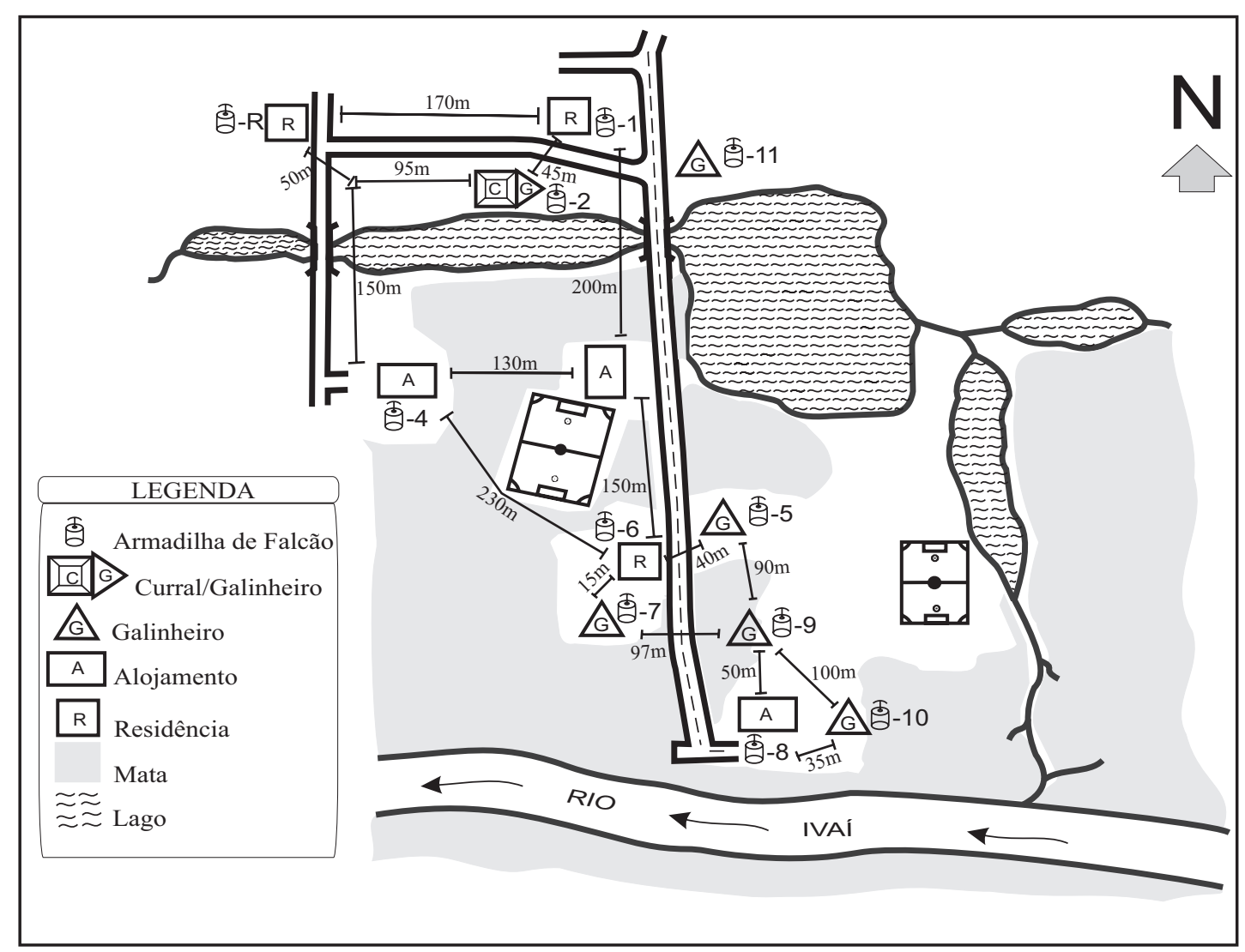

Figura 1 - Distribuição das armadilbas de Falcão nos diversos ecótopos no Recanto Marista, Município de Doutor Camargo, Paraná, de maio de 2005 a abril de 2006. 
- Ecótopo 1 (E1): na varanda da residência do caseiro, na entrada do Recanto Marista.

- Ecótopo 2 (E2): dentro de um galinheiro, ao lado do qual foi construído um curral em janeiro de 2006, que abriga ovinos e, eventualmente, bovinos.

- Ecótopo R (R): nos fundos de uma residência que é, eventualmente, alugada para visitantes.

- Ecótopo 4 (E4): num anexo ao lado de uma residência onde eventualmente são alojados trabalhadores temporários.

- Ecótopo 5 (E5): num galinheiro a uma distância de $40 \mathrm{~m} \mathrm{da}$ residência do administrador.

- Ecótopo 6 (E6): na varanda da residência do administrador.

- Ecótopo 7 (E7): num galinheiro distante 15 metros da casa do administrador.

- Ecótopo 8 (E8): na entrada principal de um alojamento, às margens do rio Ivaí, usado por grupos de pessoas que procuram retiro espiritual.

- Ecótopo 9 (E9): num galinheiro atrás do alojamento.

- Ecótopo 10 (E10): num galinheiro ao lado direito do alojamento, dentro da mata.

- Ecótopo 11 (E11): num galinheiro, próximo à cancela, na entrada do Recanto Marista, construído em junho de 2002, com a finalidade de atrair flebotomíneos do ecótopo 1.

0 acompanhamento de medidas para diminuir a população desses insetos no domicílio vem sendo feito desde $1998^{28}$. Tais medidas constam de: a) retirada de folhas, frutos, resíduos agrícolas, fezes de animais domésticos e restos de alimentos a eles oferecidos e outros tipos de matéria orgânica do solo; b) evitar a umidade do solo, no peridomicílio, dando um destino adequado para as águas de uso doméstico. A partir do momento que 0 acompanhamento foi instituído, vetou-se a permanência de cães no Recanto Marista. Além das medidas preconizadas, todas as edificações (residências, silos e outras) e abrigos de animais domésticos (galinheiros, currais, etc) foram desinsetizados: com deltametrina $(250 \mathrm{mg} / \mathrm{L})$ em junho de 2005 , nos galinheiros, e setembro de 2005, no alojamento e nos banheiros próximos à quadra de esportes; com malation $(1 \mathrm{~g} / \mathrm{L})$ em fevereiro de 2006, em todos os ambientes.

Estipulou-se que de 3 a 5 galinhas seriam mantidas em cada galinheiro (ecótopos E2, E5, E7, E9, E10, E11) como fator de atração para os flebotomíneos.

Os resultados de uma pesquisa realizada anteriormente no Recanto Marista, de abril de 2001 a setembro de $2002^{25}$, são parcialmente resgatados na Tabela 2, para a comparação com os dados obtidos nesta pesquisa. Os ecótopos utilizados para as coletas foram os mesmos, exceto o ecótopo 3 (E3), que era representado por um curral construído próximo da residência (R) e que foi desativado.

Os flebotomíneos foram preparados e identificados no Laboratório de Parasitologia Básica da UEM. A nomenclatura segue Galati ${ }^{11}$.
Para comparações da frequiência das espécies em relação aos ecótopos foi utilizado o teste não-paramétrico de Kruskal-Wallis. Para a comparação dos períodos de captura de 2001/2002 e 2005/2006, foi usado o teste de Wilcoxon por Rank Médio. Para a comparação entre os totais de flebotomíneos coletados nos galinheiros (E2, E5, E7, E9, E10 e E11) e os demais ecótopos utilizou-se o teste Estatístico de Mann-Whitney. Para a realização dos testes foi utilizado o software Statistica 6.0 considerando um nível de significância de 5\%. Para o cálculo dos valores em logaritmos da Figura 3, às quantidades de exemplares das espécies Nyssomyia neivai e Nyssomyia whitmani foi adicionada uma unidade.

\section{RESULTADOS}

Na Tabela 1, verifica-se que foram coletadas as seguintes espécies de flebotomíneos: Brumptomyia brumpti (Larrousse), Brumptomyia cunbai (Mangabeira), Evandromyia cortelezzii (Brèthes), Evandromyia correalimai (Martins, Coutinho \& Luz), Expapillata firmatoi (Barretto, Martins \& Pellegrino), Micropygomyia ferreirana (Barreto, Martins \& Pellegrino), Migonemyia migonei (França), Nyssomyia neivai (Pinto), Nyssomyia whitmani (Antunes \& Coutinho), Pintomyia fischeri (Pinto), Pintomyia monticola (Costa Lima), Pintomyia pessoai (Coutinho \& Barreto), Psathyromyia aragaoi (Costa Lima), Psathyromyia shannoni (Dyar) e Sciopemyia sordellii (Shannon \& Del Ponte). Foram coletados 213.195 flebotomíneos, sendo 149.296 fêmeas e 63.899 machos. Do total de flebotomíneos, $160.785(75,4 \%)$ foram exemplares de Nyssomyia neivai que juntamente com as espécies de Nyssomyia whitmani $(23,4 \%)$, Migonemyia migonei $(0,8 \%)$ e Pintomyia fischeri $(0,4 \%)$, somaram $212.532(99,7 \%)$ do total de flebotomíneos coletados.

As variações entre os totais de insetos capturados nos diferentes ecótopos eram muito grandes, mas os de E1 eE5, E1 eE10, E5 eE10, E7 eE9 $(p>0,05)$ não foram estatisticamente significantes (teste de Kruskal-Wallis). 0 teste mostra também que houve diferença entre as quantidades de exemplares de Nyssomyia whitmani coletados na maioria dos ecótopos, com exceção de E1 e E9, E1 e E10, R e E4, E4 e E6, E5 e E8, E9 e E10 (p> 0,05). 0 mesmo teste ainda mostra que houve diferença entre as quantidades de exemplares de Nyssomyia neivai coletados na maioria dos ecótopos, exceto E1 e E10, E2 e E9, E5 e E7, E7 e E9 (p> 0,05) (Tabela 1).

Quando se compara o número de flebotomíneos capturados nos galinheiros (ecótopos E2, E5, E7, E9, E10 e E11), com aquele dos demais ecótopos (E1, R, E4, E6 e E8), observa-se que o número de espécimes coletados nos primeiros (189.124; 88,7\%) foi significativamente maior que nos segundos $(24.071 ; 11,3 \%)$ $(\mathrm{p}<0,0176)$, conforme o teste de Mann-Whitney (Tabela 1$)$.

Verifica-se na Tabela 2 que no ecótopo E11 obteve-se a maior média horária de flebotomíneos $(\mathrm{MH}=379,5)$, seguindo-se os ecótopos E10 $(\mathrm{MH}=270,0)$, E9 $(\mathrm{MH}=154,0)$ e E2 $(\mathrm{MH}=88,6)$, no período de realização deste trabalho. 0 teste por Rank Médio para grupos pareados de Wilcoxon mostra que existe diferença estatística entre as médias horárias de flebotomíneos coletados nos períodos de 2001/2002 e $2005 / 2006(\mathrm{p}=0,0051)$. 
Tabela 1 - Flebotomíneos coletados em diferentes ecótopos no Recanto Marista, Município de Doutor Camargo, Paraná, de maio de 2005 a abril de 2006.

\begin{tabular}{|c|c|c|c|c|c|c|c|c|c|c|c|c|}
\hline Espécie/Ecótopo & $\mathrm{E}^{2 * *}$ & $\mathrm{E} 2 *$ & $\mathrm{R}^{* * *}$ & $\mathrm{E} 4^{* * *}$ & $\mathrm{E} 5^{*}$ & $\mathrm{E} 6^{* * *}$ & $\mathrm{E} 7 *$ & $\mathrm{E} 8 * *$ & E9* & E10* & E11* & Total \\
\hline Nyssomyia neivai $^{\text {a }}$ & 7.523 & 12.263 & 1.058 & 1.824 & 6.139 & 3.398 & 11.120 & 6.851 & 23.295 & 42.274 & 45.040 & 160.785 \\
\hline Nyssomyia whitman ${ }^{\text {b }}$ & 1.636 & 4.519 & 124 & 222 & 302 & 246 & 830 & 907 & 5.228 & 8.215 & 27.003 & 49.232 \\
\hline Migonemyia migonei & 40 & 144 & 3 & 34 & 8 & 11 & 34 & 49 & 222 & 776 & 309 & 1.630 \\
\hline Pintomyia fischeri & 25 & 25 & 4 & 31 & 10 & 8 & 23 & 30 & 88 & 301 & 340 & 885 \\
\hline Pintomyia pessoai & 5 & 48 & 1 & 2 & 5 & 1 & 12 & 15 & 82 & 105 & 122 & 398 \\
\hline Pintomyia monticola & - & 4 & - & 4 & 1 & - & - & 3 & 10 & 14 & 10 & 46 \\
\hline Psathyromyia shannoni & - & 5 & - & - & 16 & - & 1 & 2 & 12 & 43 & 16 & 95 \\
\hline Expapillata firmato $i$ & - & 3 & - & - & 3 & - & 2 & 2 & 5 & 21 & 3 & 39 \\
\hline Evandromyia cortelezzii & - & 2 & - & 1 & 3 & 1 & 2 & 3 & 7 & 13 & 1 & 33 \\
\hline Brumptomyia brumpti & - & 4 & - & - & - & 1 & 1 & 4 & 7 & 14 & 12 & 43 \\
\hline Brumptomyia cunhai & - & - & - & - & - & - & - & 1 & 1 & - & 1 & 3 \\
\hline Psathyromyia aragao $i$ & - & - & - & - & - & - & - & - & - & - & 1 & 1 \\
\hline Micropygomyia ferreirana & 1 & - & - & - & - & - & - & - & - & 2 & - & 3 \\
\hline Sciopemyia sordellii & - & - & - & - & - & - & - & - & - & 1 & - & 1 \\
\hline Evandromyia correalimai & - & 1 & - & - & - & - & - & - & - & - & - & 1 \\
\hline Total $^{c}$ & 9.230 & 17.018 & 1.190 & 2.118 & 6.487 & 3.666 & 12.025 & 7.867 & 28.957 & 51.779 & 72.858 & 213.195 \\
\hline Porcentagem & 4,3 & 8,0 & 0,6 & 1,0 & 3,0 & 1,7 & 5,6 & 3,7 & 13,6 & 24,3 & 34,2 & 100,0 \\
\hline
\end{tabular}

Localização das armadilhas: *Galinheiros. ***outros ecótopos.

Teste de Kruskal-Wallis mostra que: a. houve diferença entre as quantidades de exemplares de $N$. neivai coletados na maioria dos ecótopos, exceto E1 e E10, E2 e E9, E5 e E7, E7 e E9 (p> 0,05); b. houve diferença entre as quantidades de exemplares de $N$. whitmani coletados na maioria dos ecótopos, exceto entre E1 e E9, E1 e E10, R e E4, E4 e E6, E5 e E8, E9 e E10 (p>0,005); c. houve diferença entre as quantidades totais de espécimes coletados na maioria dos ecótopos, exceto entre E1 e E5, E1 e E10, E5 e E10, E7 e E9 (p > 0,05)

Teste de Mann-Witney: o número de exemplares coletados nos galinheiros* [E2, E5, E7, E9, E10 e E11] foi maior do que o coletado nos demais ecótopos*** [E1, R, E4, E6 e E8] $(\mathrm{p}<0,0176)$.

Tabela 2 - Distribuição e média horária (MH) de flebotomíneos coletados por ecótopo no Recanto Marista, município de Doutor Camargo, Paraná, de março de 2001 a setembro de 2002 e de maio de 2005 a abril de 2006.

\begin{tabular}{|c|c|c|c|c|c|}
\hline \multicolumn{3}{|c|}{$2001 / 2002^{25}$} & \multicolumn{3}{|c|}{$2005 / 2006$} \\
\hline Ecótopo & Total & MH & Ecótopo & Total & $\mathrm{MH}$ \\
\hline E1 & 18.412 & $121,1^{*}$ & E1 & 9.230 & $48,1^{*}$ \\
\hline E2 & 14.870 & $97,8^{*}$ & E2 & 17.018 & $88,6^{*}$ \\
\hline $\mathrm{R}$ & 2.021 & $13,3^{*}$ & $\mathrm{R}$ & 1.190 & $6,2^{*}$ \\
\hline E3 & 1.756 & 11,6 & - & - & - \\
\hline E4 & 3.543 & $23,3^{*}$ & E4 & 2.118 & $11,0^{*}$ \\
\hline E5 & 6.948 & $45,7^{*}$ & E5 & 6.487 & $33,7^{*}$ \\
\hline E6 & 4.317 & $28,4^{*}$ & E6 & 3.666 & $19,1^{*}$ \\
\hline E7 & 14.265 & $93,8^{*}$ & E7 & 12.025 & $62,6^{*}$ \\
\hline E8 & - & - & E8 & 7.867 & 41,0 \\
\hline E9 & 26.653 & $175,3^{*}$ & E9 & 28.957 & $154,0^{*}$ \\
\hline E10 & 95.404 & $627,6^{*}$ & E10 & 51.779 & $270,0^{*}$ \\
\hline E11 & 11.632 & $415,4^{*}$ & E11 & 72.858 & $379,5^{*}$ \\
\hline Total & 199.821 & $1.653,5$ & Total & 213.195 & $1.113,8$ \\
\hline
\end{tabular}

Localização das armadilhas: E1: varanda/residência; E2: galinheiro; E3: curral atualmente desativado; R: fundos/casa; E4: anexo/casa; E5: galinheiro; E6: varanda/ casa; E7: galinheiro; E8: alojamento; E9: galinheiro; E10: galinheiro; E11: galinheiro. *Teste de Wilcoxon por Rank Médio ( $\mathrm{p}=0,0051)$ : existe diferença estatística entre as médias horárias $(\mathrm{MH})$ de flebotomíneos coletados nos dois períodos.

Na Figura 2, nota-se que, no total, os picos do número de flebotomíneos ocorreram nos meses de março, abril, novembro e dezembro, coincidindo com os picos de pluviosidade e com temperaturas elevadas. De um modo geral, o mesmo fato ocorreu em cada um dos ecótopos, no tocante às espécies Nyssomyia whitmani $\mathrm{e}$ Nyssomyia neivai (Figura3). Nota-se ainda que no período de coletas os meses de maior precipitação de chuva foram fevereiro, março, setembro, outubro e novembro e o mês mais seco foi agosto.
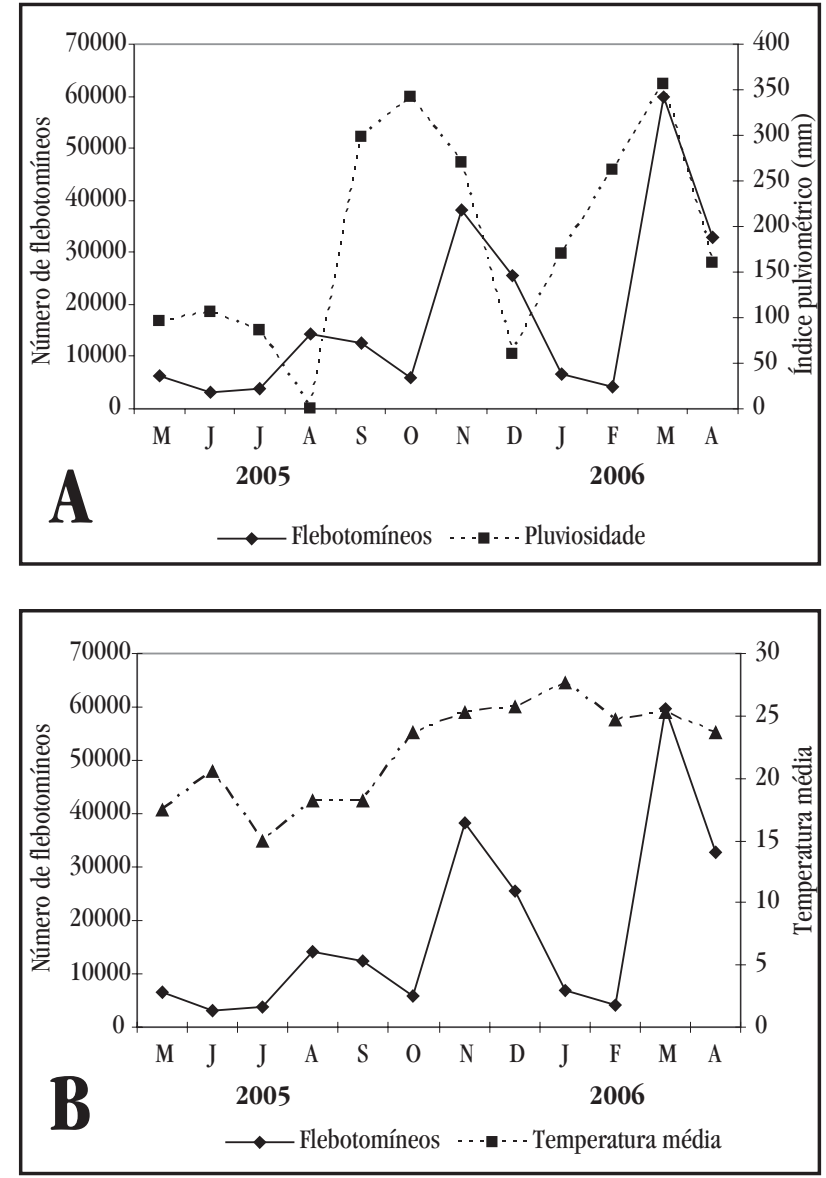

Figura 2 - Sazonalidade de flebotomíneos no conjunto das armadilhas instaladas nos diversos ecótopos no Recanto Marista, precipitações pluviométricas mensais (A) e temperaturas médias (B), no Município de Doutor Camargo, Paraná, de maio de 2005 a abril de 2006. 


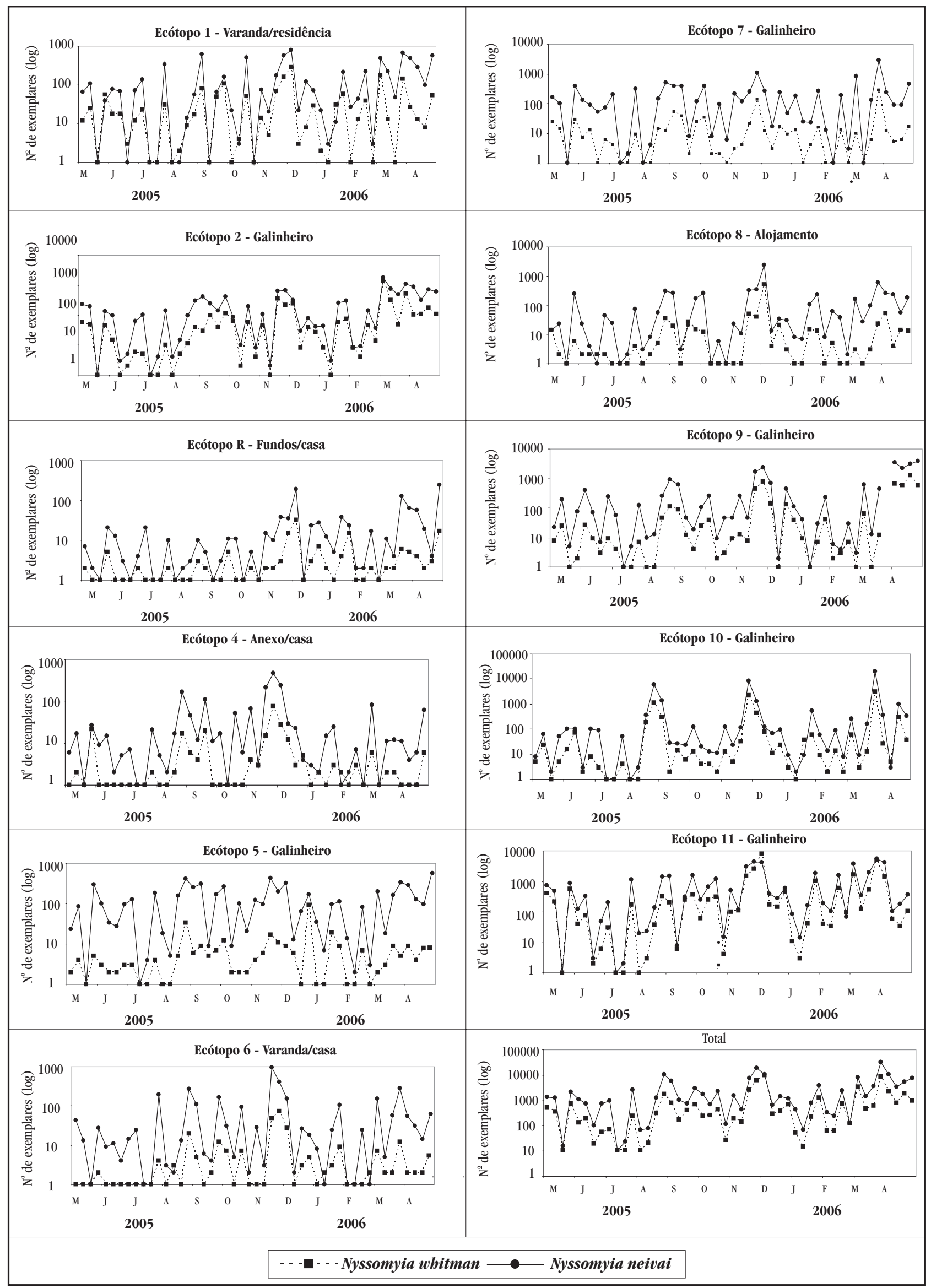

Figura 3 - Sazonalidade de Nyssomyia whitmani e Nyssomyia neivai nos diversos ecótopos no Recanto Marista, Município de Doutor Camargo, de maio de 2005 a abril de 2006. 


\section{DISCUSSÃO}

As espécies coletadas já foram descritas anteriormente no Recanto Marista, exceto Migonemyia aragaoi e Sciopemyia sordelli $^{27}{ }^{28}$. Nesta localidade, tem predominado Nyssomyia neivai ${ }^{2728}$. As espécies Nyssomyia neivai, Nyssomyia whitmani, Migonemyia migonei, Pintomyia fischeri e Pintomyia pessoai têm sido constantes no Recanto Marista e em outras localidades, no Estado do Paraná ${ }^{17} 29$

As espécies Nyssomyia neivai e Nyssomyia intermedia ocorrem em matas e se adaptam muito bem às suas bordas e nos ambientes modificados, podendo se desenvolver no peridomicílio e invadir o domicílio ${ }^{16}$. Nyssomyia intermedia e Nyssomyia whitmani têm sido incriminadas como vetoras dos parasitos da LTA ${ }^{15}$. Embora Nyssomyia neivai ainda não tenha sido encontrada com infecção natural por Leishmania, possivelmente essa espécie esteja envolvida na epidemiologia desta zoonose no Paraná, uma vez que ela é filogeneticamente próxima de Nyssomyia intermedia ${ }^{5}$. Nyssomyia whitmani, a segunda espécie mais abundante no Recanto Marista, é bastante antropofílica e bem adaptada aos ambientes florestais, com tendência a rarear onde ocorre desmatamento ${ }^{16}$.

Verificou-se que o número de flebotomíneos coletados nos ecótopos representados pelos galinheiros $(88,7 \%)$ foi significativamente maior do que o coletado nos demais ecótopos. Quando se compara os ecótopos mais próximos uns dos outros (E1, E2, E11) (E5, E6, E7) (E8, E9, E10) (Figura 1) verifica-se que: a) os flebotomíneos coletados nos ecótopos E2 e E11 (galinheiros) foram significativamente superiores aos coletados no ecótopo E1 (residência); b) nos ecótopos E5 e E7 (galinheiros) eles foram superiores aos coletados no E6 (residência); c) e nos ecótopos E9 e E10 superiores aos coletados no E8 (alojamento). 0 que sugere que os galinheiros podem estar atuando como barreiras zooprofiláticas, diminuindo a população de flebotomíneos nos domicílios e no alojamento existentes no Recanto Marista. Resultados semelhantes foram assinalados anteriormente nesta localidade ${ }^{25} 2728$ e no município de Jussara $^{23}{ }^{29}$. Entretanto, Alexander e cols ${ }^{3}$ questionam a criação de galinhas e de outros animais domésticos, nos quintais de moradores da periferia de grandes cidades, como um fator que pode influenciar no risco de leishmaniose visceral em humanos. Contudo, os mesmos autores ressaltam ainda o fato de que a alimentação dos flebotomíneos com o sangue de aves pode impedir a manutenção da infecção por Leishmania nesses insetos, além de que uma segunda alimentação do mesmo tipo pode eliminar a infecção. Tudo indica que as desinsetizações não surtiram efeitos, pois nos meses subseqüentes às mesmas não houve diminuição da população de flebotomíneos nos ecótopos desinsetizados.

No transcurso deste trabalho, o número de galinhas nos galinheiros foi de três a cinco, exceto no ecótopo 2, onde havia apenas uma galinha a partir de janeiro de 2006. Coincidentemente, ocorreram problemas na instalação elétrica do galinheiro (E2) e até o final das coletas a lâmpada desse ecótopo permaneceu desligada. Além disso, cinco ovinos e ocasionalmente três bovinos foram abrigados ao lado do ecótopo 2 . Acredita-se que o número de flebotomíneos coletados nesse ecótopo tenha sido elevado nos meses de março e abril devido à atração exercida pela permanência desses animais ao lado desse ecótopo, pois os animais domésticos em maior número atraem esses dípteros também em maior número do que uma fonte luminosa ${ }^{17} 22$.

Houve uma redução da MH de flebotomíneos coletados em relação às $\mathrm{MH}$ de trabalhos anteriormente realizados no Recanto Marista ${ }^{25} 2728$. É importante ressaltar que em 1996 foram notificados 10 casos de leishmaniose entre os 24 moradores dessa localidade, além de nove cães com sorologia positiva ${ }^{27}$. As medidas para o controle da população de flebotomíneos foram implantadas após a primeira pesquisa ${ }^{27}$, contudo a sua execução não tem sido rigorosa. Daí talvez a ocorrência de quatro casos de LTA no Recanto Marista, um em 2001, um em 2004 e dois em 2005. Os dois primeiros casos (duas mulheres adultas) eram representados por uma moradora da localidade e o outro por uma visitante. Os outros dois casos eram de trabalhadores temporários (de agosto a dezembro de 2005), que, apesar de avisados do risco, pescavam freqüentemente no período noturno. Apesar das medidas de controle de flebotomíneos não terem sido seguidas de forma rigorosa, não houve mais surto epidêmico de LTA no Recanto Marista.

Ao comparar a sazonalidade dos flebotomíneos coletados no conjunto das armadilhas com as precipitações pluviométricas mensais e com as temperaturas médias mensais, percebe-se que a quantidade de flebotomíneos coletados recrudesce após as precipitações de chuva e temperaturas elevadas, confirmando os dados assinalados em outros trabalhos ${ }^{2024}$. Chama a atenção um discreto pico no número de flebotomíneos coletados no mês de agosto. Apesar do baixo índice pluviométrico neste mês, ocorreu uma pequena elevação na temperatura em relação ao mês de julho. Esta situação já foi assinalada no Paraná ${ }^{20}$.

Os gráficos da Figura 3, relativos a cada um dos ecótopos, mostram que o comportamento sazonal de Nyssomyia whitmani e Nyssomyia neivai é semelhante, quando analisados no conjunto. Porém, em ecótopos localizados próximos um dos outros (E1, E2 e E11; E5, E6 e E7; E8, E9 e E10), a semelhança do comportamento sazonal é mais evidente.

As diferenças entre as proporções de Nyssomyia neivai e Nyssomyia whitmani aumentaram de 79,2\% e 18,2\% (1996/1997) para 88,4\% e 11,1\% (1998/2000) ${ }^{27}$ e 90,4\% e 8,5\% $(2001 / 2002)^{25}$, diminuindo no período de coletas deste trabalho (75,4\% e 23,4\%). Nos três primeiros períodos, a mudança nas proporções das referidas espécies ocorreu, possivelmente, devido ao impacto das alterações introduzidas no meio ambiente, visto que Nyssomyia neivai se adapta melhor nos ambientes com alto grau de antropia. Contudo, a diminuição da diferença entre as proporções de Nyssomyia neivai e Nyssomyia whitmani, no último período de coletas pode ter sofrido a influência da localização dos ecótopos, pois a proporção de Nyssomyia neivai nos ecótopos E1, E2, R, E11 foi de 66,4\% e de Nyssomyia whitmani foi de 33,6\%. Nos demais ecótopos a proporção de Nyssomyia neivai foi de 85,6\% e a de Nyssomyia whitmani foi de $14,4 \%$. Portanto, a diferença entre as proporções de Nyssomyia 
neivai e Nyssomyia whitmani foi menor no primeiro conjunto de ecótopos, que se encontra em área de latossolo vermelho e vermelho-amarelo ou nitossolo, que retêm mais umidade. Nos demais ecótopos, que se encontram em área de solo mais arenoso, bem drenado e derivado do arenito Caiuá, a diferença entre as proporções dessas espécies foi maior. Em outras localidades do Paraná, no primeiro tipo de solo tem predominado Nyssomyia whitmani, enquanto no segundo tem predominado Nyssomyia neivai $^{17} 26$.

Assim, constata-se que: 1) as médias horárias de flebotomíneos foram menores em todos os ecótopos em relação às médias de coletas realizadas em períodos anteriores; 2 ) a maioria (88,7\%) dos flebotomíneos foi coletada nos ecótopos representados por galinheiros, reforçando a hipótese de que eles podem atuar como barreiras zooprofiláticas; 3) houve uma redução acentuada no número de casos humanos de LTA, a partir do momento que as medidas profiláticas foram introduzidas; 4) entre as duas espécies dominantes no Recanto Marista, Nyssomyia neivai e Nyssomyia whitmani, somente a última tem comprovada importância na epidemiologia da LTA; 5) considerando que a primeira espécie tem prevalecido e mantido uma densidade populacional elevada nesse recanto, sua importância na transmissão de Leishmania não deve ser subestimada.

\section{AGRADECIMENTOS}

Ao Colégio Marista, pelo apoio logístico; à Dra. Eunice Aparecida Bianchi Galati, da Faculdade de Saúde Pública/USP, pelo auxílio na identificação de flebotomíneos; ao Sr. Walmir Schreiner, engenheiro agrônomo da Cooperativa Agroindustrial (COCAMAR), de Doutor Camargo, pelos dados pluviométricos; à mestranda Janaína Sales de Freitas, do Departamento de Análises Clínicas, e ao João Eduardo Cavalcanti Brito, do curso de Biologia da UEM, pelo auxílio nas coletas e na identificação de flebotomíneos.

\section{REFERÊNCIAS}

1. Alencar JE. Expansão do calazar no Brasil. Ceará Médico 5: 86-102 1983.

2. Alencar JE. Profilaxia do calazar no Brasil no Ceará, Brasil. Revista do Instituto de Medicina Tropical de São Paulo 3: 175-180 1961.

3. Alexander B, Carvalho RL, McCallum H, Pereira MH. Domestic chicken (Gallus gallus) in the epidemiology of urban visceral leishmaniasis in Brazil. Emerging Infectious Diseases [serial on line] $2002 \mathrm{Dec} ; 8$. Available from: URL: http://www. cdc.gov/ncidod/EID/vol8no12/01-0485.htm [03/05/2005], 2002.

4. Alexander B, Maroli M. Control of Phlebotomine Sandflies. Medical Veterinary Entomological 17: 1-18, 2003.

5. Andrade Filho JD, Galati EAB, Falcão AL. Redescription of Nyssomyia intermedia (Lutz \& Neiva, 1912) and Nyssomyia neivai (Pinto, 1926) (Diptera: Psychodidae). Memórias do Instituto Oswaldo Cruz 98: 1059-1065, 2003.

6. Campbell-Lendrum D, Dujardin JP, Martinez E, Feliciangeli MD, Perez JE, Silans LNMP, Desjeux P. Domestic and Peridomestic Transmission of American Cutaneous Leishmaniasis: Changing Epidemiological Patterns Present New Control Opportunities. Memórias do Instituto Oswaldo Cruz 96: 159-162, 2001.

7. Davies CR, Llanos-Cuentas EA, Campos P, Monge J, Leon E, Canales J. Spraying haouses in th Peruvian Andes with lambdacyhalothrin protects residents against cutaneous leishmaniasis. Transactions of the Royal Society of Tropical Medicine and Hygiene 94: 631-636, 2000.

8. Deane LM, Deane MP, Alencar JE. Observações sobre o combate ao Phlebotomus longipalpis pela desinsetização domiciliária, em focos endêmicos de calazar no Ceará. Revista Brasileira de Malariologia e Doenças Tropicais 7: 131-141, 1955.

9. Desjeux P. The increase in risk factors for leishmaniasis worldwide. Transactions of the Royal Society of Tropical Medicine and Hygiene 95: 239-243, 2001.

10. Feliciangeli MD, Maroli M, Wheeler A, Townson H, Ward R, Maignon R. Sandfly control trial with deltamethrin impregnated curtains in El Ingenio, Miranda State, Venezuela. Boletin de la Direccion de Malariologia y Saneamiento Ambiental 35: 127-132, 1995.

11. Galati EAB. Morfologia e Taxonomia. In: Rangel EF, Lainson R (eds) Flebotomíneos do Brasil. Editora da Fundação Oswaldo Cruz, Rio de Janeiro, 2003.

12. Gratz NG. Emerging and Resurging Vector-Borne Diseases. Annual Review of Entomology 44: 51-75, 1999.

13. Kelly DW, Mustafa Z, Dye C. Differential application of lambdacyhalothrin to control the sandfly Lutzomyia longipalpis. Medical Veterinary Entomological 11: 23-24, 1997.

14. Kroeger A, Avila EV, Morison L. Insecticide impregnated curtains to control domestic transmission of cutaneous leishmaniasis in Venezuela: cluster randomised trial. British Medical Journal 325: 810-813, 2002.

15. Luz E, Membrive N, Castro EA, Dereure J, Pratlong F, Dedet A, Pandev A, Thomaz-Soccol V. Lutzomyia whitmani (Diptera: Psychodidae) as vector of Leishmania (V.) braziliensis in Paraná State, Southern Brazil. Annals of Tropical Medicine and Parasitology 94: 623-631, 2000.

16. Marcondes CB. Flebotomíneos. In: Marcondes CB (eds) Entomologia Médica e Veterinária. Editora Atheneu, São Paulo, Rio de Janeiro, Belo Horizonte, 2001.

17. Membrive NA, Rodrigues G, Membrive U, Monteiro WM, Neitzke HC, Lonardoni MVC, Silveira TGV, Teodoro U. Flebotomíneos de municípios do Norte do Estado do Paraná, sul do Brasil. Entomología y Vectores 11: 673-680, 2004.

18. Ministério da Saúde. Leishmaniose Tegumentar Americana - Distribuição de casos confirmados de LTA de 1980 a 2005. http://dtr2001.saude.gov.br/svs/epi/ situacao_doencas/planilhas_doencas.htm. Acessado em 12/12/2006.

19. Silva AC, Gomes AC. Estudo da competência vetorial de Lutzomyia intermedia (Lutz \& Neiva, 1912) para Leishmania (Viannia) braziliensis, Vianna, 1911. Revista da Sociedade Brasileira de Medicina Tropical 34: 187-191, 2001.

20. Teodoro U, Alberton D, Kühl JB, Santos ES, Santos DR, Santos AR, Oliveira O, Silveira TGV, Lonardoni MVC. Ecologia de Lutzomyia (Nyssomyia) whitmani (Antunes \& Coutinho, 1939) (Diptera, Psychodiade) em área urbana no sul do Brasil. Revista de Saúde Pública 37: 651-656, 2003 a.

21. Teodoro U, Galati EAB, Kühl JB, Lozovei AL, Barbosa OC. Controle de flebotomíneos com DDT, em área endêmica de leishmaniose tegumentar no Estado do Paraná, sul do Brasil. Brazilian Archives of Biology and Technology 41: 359-364, 1998 .

22. Teodoro U, Kühl JB, Abbas M, Dias AC. Luz e de aves como atrativos de flebotomíneos (Diptera, Psychodidae), no sul do Brasil. Revista Brasileira de Entomologia 45: 167-172, 2001a.

23. Teodoro U, Kühl JB, Thomaz-Soccol V, Barbosa 0C, Ferreira MEMC, Lozovei AL, Versignassi TG, Roberto ACBS. Environmental sanitation and peridomiciliar organization as auxiliary practices for the control of phlebotomines in Paraná State, Southern Brazil. Brazilian Archives of Biology and Technology 42: 307-314, 1999.

24. Teodoro U, La Salvia Filho V, Lima EM, Spinosa RP, Barbosa OC, Ferreira MEMC, Lonardoni MVC. Observações sobre o comportamento de flebotomíneos em ecótopos florestais e extraflorestais, em área endêmica de leishmaniose tegumentar americana, no norte do Estado do Paraná, sul do Brasil. Revista de Saúde Pública 27: 242-249, 1993.

25. Teodoro U, Santos DR, Santos AR, Oliveira O, Poiani LP, Kühl JB, Lonardoni MVC, Silveira TGV, Monteiro WM, Neitzke HC. Avaliação de medidas de controle de flebotomíneos no norte do Estado do Paraná, Brasil. Cadernos de Saúde Pública 23: 2597-2604, 2007. 
26. Teodoro U, Santos DR, Santos AR, Oliveira 0, Poiani LP, Silva AM, Neitzke HC, Monteiro WM, Lonardoni MVC, Silveira TGV. Informações Preliminares sobre Flebotomíneos do Norte do Paraná. Revista de Saúde Pública 40: 327-330, 2006

27. Teodoro U, Silveira TGV, Santos DR, Santos ES, Santos AR, Oliveira O, Kühl JB. Freqüência da fauna de flebotomíneos no domicílio e em abrigos de animais domésticos no peridomicílio, nos municípios de Cianorte e Doutor Camargo, Estado do Paraná, Brasil. Revista de Patologia Tropical 30: 209-223, 2001b.
28. Teodoro U, Silveira TGV, Santos DR, Santos ES, Santos AR, Oliveira 0, Kühl JB, Alberton D. Influência da reorganização, da limpeza do peridomicílio e da desinsetização de edificações na densidade populacional de flebotomíneos, no município de Doutor Camargo, Estado do Paraná, Brasil. Cadernos de Saúde Pública 19: 1801-1813, 2003b.

29. Teodoro U, Thomaz-Soccol V, Kühl JB, Santos DR, Santos ES, Santos AR, Abbas M, Dias AC. Reorganization and Cleaness of Peridomiciliar Area to Control Sand flies (Diptera, Pschodidae, Phlebotominae) in South Brazil. Brazilian Archives of Biology and Technology 47: 205-212, 2004 